\title{
Review of Stellite Alloys Intelligent Processing Methods in China
}

\author{
Xiao-Guang Yue, Zhi-Gang Liu and Xiao-Zhi Wang
}

Nanchang Institute of Science and Technology, Nanchang 330108, China

\begin{abstract}
Keywords: Stellite alloys, Intelligent processing, China.
\end{abstract}
\begin{abstract}
Stellite is cemented carbide to resist a variety of wear and corrosion and high temperature oxidation. This paper introduces a series of domestic Stellite alloy intelligent processing methods. In China, Stellite alloy academic research is not very popular, but many of them have representation. These methods for the study of engineering and technology have reference value.
\end{abstract}

\section{Introduction}

Stellite is a wear-resistant, corrosion-resistant, and high temperature oxidation-resistant alloy. The main component of Stellite is cobalt; and it also contains a considerable amount of nickel, chromium, tungsten, and a small amount of molybdenum, niobium, tantalum, titanium, lanthanum, and other alloying elements. Depending on the alloy composition, it can be made of wire and powder, such as hard facing, thermal spraying, and spray. It can also be used for forging and powder metallurgy.

The Stellite alloy cost is too expensive, originally only used in the manufacture of small Stellite alloy castings, from 1992 onwards, Stellite alloy surface coating started for large parts, in order to solve the internal combustion engine and the subsequent jet engine special requirements for materials. This can slow down large parts of the surface wear and corrosion, and can save materials.

As a kind of cemented carbide, Stellite alloy is development to a certain role in promoting the world industry. In recent years, the domestic scholars have conducted a series of research on the Stellite Alloy process. The research mainly focuses on two aspects: 1 . Stellite alloy substrate surface covering technology; 2. the surface modification of forming Stellite alloy.

\section{Surface Coverage Technology}

Brazing Processing. Because the organization of Stellite alloy has high stability, high hardness, so in the water droplets under the impact will only cause a small amount of deformation, and because of the excellent toughness, not easy to crack, its hardness and toughness of two of the superiority, make the blade become better anti-corrosion material. Stellite alloy is mainly used for repair welding of the blade when it first introduced the domestic, used to improve water erosion resistance of the blade. The first is the use of domestic brazing; welding the finished products Stellite alloy slices on the edge of upper leaves of entrance side and back arc side. On the blade of brazing Stellite alloy slices can indeed play a role in preventing the blade water erosion, but this process has a great weakness. In the actual process of brazing Stellite alloy, due to the less dense of brazing will produce some inevitable shortcomings. After testing found that, there are some pores and incomplete penetration cracks exist in the surface and inside of Stellite Alloy slices; exceed the standard clearance alloy sheet; there is a sharp angle between the tall of Stellite alloy slices and base metal, it's easy to cause the stress concentration; Because of the above disadvantages, leading to water erosion ability on the blade will have a certain decline, especially in the junction, Stellite alloy slices may fall off. Brazing Stellite alloy slices produced a series of faults, basic it is due to the unreasonable process caused by imperfect. The domestic made certain exploration to directly on the blade direct brazing of optimum process parameters of Stellite Alloy slices [4], to improve the above shortcomings played a certain help.

Magnetron Sputtering Deposition. The method initially embedded directly Stellite alloy slices has inevitable shortcomings, so the domestic explore a series of new techniques to deal with Stellite 
alloy. Shen Yong and Huang Ningkang of the Sichuan University, by DC magnetron sputtering and RFDC magnetron technology for preparing a layer of Stellite alloy film on the base material [5-7]. And measured the microstructure and composition of the film. For alloy sputtering, the most important problem is whether the prepared deposition layer composition meets the requirements. Composition study show that by magnetron sputtering technique preparation Stellite Alloy Thin Films is very uniform and meet the standard. Comparing the two kinds of magnetron sputtering technology, when acquire the similar performance thin film; use DC magnetron sputtering deposit Stellite alloy will greatly reduce the equipment investment, easy for operation and maintenance. This provides a favorable way for this technology into industrial application. Magnetron sputtering deposition of Stellite alloy has the advantage of fast speed, low base material temperature and has small damage to the film. Stellite film and base material combination well, the process is easy to repeat, can get uniform film in large area on the base material, easy to realize industrialization. So till now, the domestic use magnetron sputtering technique to prepare the Stellite multiple layers.

Plasma Arc Surfacing. Plasma arc surfacing technique is a branch of material surface modification, it uses the plasma arc as the heat source, and let alloy powder cladding on the surface of the work piece is used to improve the wear resistance and corrosion resistance of materials. In the process of plasma arc surfing Stellite, dilution rate is the most attention index. The dilution rate increase will cause the substrate metal melting quantity too much, and the performances of surfacing layer reduce serious. The production cost is increased, the efficiency will decline. Zhu Kai from Jiangsu University of Science and Technology to adopt this method in Q235 steel cladding Stellite alloy, by optimizing the parameters in order to explore the lowest dilution rate of surfacing welding layer parameters. During this process he obtains the most significant effect parameters [8]. The Stellite composite layer prepared by this method has low dilution rate, high deposition rate and the great quality [9].

\section{Surface Treatment Technology}

Laser Cladding. Laser surface treatment is a main means for high density surface treatment technology. Under certain conditions, it has the characteristics of traditional surface treatment technology and other high energy density surface treatment technology cannot reach or hard to reach [10]. Laser cladding can form wear resistance, corrosion resistance, erosion resistance and high temperature oxidation resistance of surface hardening layer. Sichuan university 720 blade substrate surface laser cladding department too stand alloy made some exploration to the rationality of the cladding. Use the plasma spray process plating the 6\# Stellite on the base material. Changing the laser density in experiment, and they found the best laser density parameter. When use the optimum laser density, Stellite composite layer will form a liquid flow and wetting base material. And the coating layer formed at this time is very smooth. After testing found the coating layer is more hardness than the base material, it can have a good effect to protect the base material. When the laser density is low, it can't form a liquid flow to moisten the substrate, or the formation of liquid flow but coated surface is rough. When the laser density is high, the base material may melt, lead to the dilution rate increased and reduce the surface hardness. Series of studies shows that use laser cladding Stellite is feasible [11-13]. The density and heating depth is adjustable, and it can focus on irregular parts. Power density of laser processing, the heating depth is adjustable, and can focus on irregular parts, has a huge advantage for fine processing. Although the Stellite 6\# had good comprehensive properties, but in many cases still cannot meet the requirements. Need to join the WC in the Stellite Alloy 6\# in order to get better strengthening effect. Zhong Minlin of Tsinghua University using double drum powder feeder respectively conveying Stellite alloy 6\# and WC , in the laser cladding process change the amount of WC, in order to find the best WC parameter which both can raise the of Stellite Alloy 6\# performance but does not cause crack [14-15]. For any cladding process, the heating to the cooling will produce a shrinkage stress. But because the laser cladding processing casting area is small, the transition region is small so that has a small shrinkage. In the process of material shrinkage contraction force is not enough to make the whole body deformation. But the shrinkage stresses still 
exist, and will be released into the melting zone and transition zone. Although the ductility of Stellite $6 \#$ very good, not like some alloy after the laser cladding at the casting zone will have some cracks. But because of the laser cladding process of heating and cooling very fast, the size of the generated transition zone is small; it will cause this regional have high stress.

Even the various parameters have reached the optimal case; cladding is still no uniform, due to reduce the cladding properties of Stellite Alloy [16]. So the laser cladding of Stellite Alloy because of its restrictions on technology, In large areas and large thickness of cladding hardness are uneven It is difficult to obtain a large area of cladding layer with perfect effect.

Electron Beam Melting and Solidifying. When use electron beam melting and solidifying, the electron beam bombardment of the surface will produce partial melting and rapid solidification to refine organization, make the best fit to hardness and toughness. And the electron beam remelting can make the chemical elements with certain alloy groups and white from the new distribution, reduce the micro segregation degree of certain elements, and improve the surface performance. In view of the domestic production of Stellite Alloy Structure coarse, water corrosion and rush is easy to destroy the crystal. Then in order to improve the organizational structure and the crystal problem of Stellite Alloy. Feng Zhirong and Huang Ningkang from the Sichuan University 720 have a series of experiments of Stellite Alloy by electron beam processing technology, and achieved certain results. Through a series of experiments and research [17-18], the use of electron beam surface treatment process for treatment of Stellite alloy clad, can obtain good metallurgical bonding coating with base material. And after processing, some chemical elements of Stellite alloy clad in re distribution, improves the corrosion resistance. The electron beam melting and solidifying, due to the process of condensation is fast, will make the crystal refinement; therefore, compared with the conventional preparation method of Stellite the hardness will be higher. But just like laser cladding. Electron beam melting also generate residual stress, but after testing, residual stresses are compressive stress, which is beneficial to improve the stress corrosion resistance and fatigue resistance of Stellite alloy. At present, the electron beam accelerating voltage up to $125 \mathrm{KV}$. Output power up to $150 \mathrm{KW}$, energy density reached $103 \mathrm{MW} / \mathrm{m} 3$. Laser can't be compared with it. So the electronic heating depth and the size are bigger than the laser, and the electron beam is good to couping with the metal surface, the utilization rate of energy is much higher than that of the laser. So the cost of the actual use of electron beam is only half of the laser processing, and processing equipment has a low price, which is about $1 / 3$ of the laser processing equipment [9]. Compared with laser cladding, the cladding microstructure uniformity is better and has low loss rate of the powder. Use electron beam to have a surface modification with stellite alloy can reduce the cost. But the electron beam is working in vacuum, this is very inconvenient. This has a certain extent limits to its application in production.

Plasma Surface Implanted. With the rapid development of surface engineering in recent years, surface strengthening technology at the core of surface engineering have increased from the hardness, strength and corrosion protection for the purpose, extended to is used to improve the stress state of work piece to adapt to a certain characteristic requirements or to extend the service life. Ion plating, plasma nitriding, ion implantation surface strengthening technology also obtained the promotion. Ion implantation is at room temperature or lower temperature and vacuum conditions make the required material ion in electric field accelerated after the bombardment of the work piece surface. This is a surface modification technology which makes ion implantation to a certain depth. Choose good ion implantation equipment and appropriate process methods and parameters, can improve the performance of many on the material like wear-resisting, corrosion resistance, oxidation resistance, hardness and fatigue strength, etc. [19-20]. In 1992 Sichuan University nuclear science and Technology Research Institute to use nitrogen ion bombardment the stellite deposited layer [21]. Experiments using Markov shape 2 cr13 stainless steel plates as base material, sputtering deposition stellite alloy, formation of the deposited film of about $100 \mathrm{~nm}$. In the experiments using different injection volume of nitrogen ion bombardment deposited film. Results showed that the nitrogen ion bombardment by department Stellite deposits can greatly improve the Markov shape 2Cr13 stainless steel substrate in corrosion resistance at $\mathrm{H} 2 \mathrm{SO} 4$ and Nacl situation. But the mechanism of corrosion 
resistance in two situations is different. As the amount of nitrogen ion bombardment injection increase to exceed the solid solubility, can form a series of such as $\mathrm{Cr} 2 \mathrm{~N}$, Fe2 $+\mathrm{nN}$ and a series of nitrides, Corrosion of - H2SO4 has played a very good role of resistance. While in Nacl solution, the amount of nitrogen ion injection increase, will consume $\mathrm{W}$, form the $\mathrm{WN}$, corrosion resistance of Nacl reduce instead. The emergence of ion implantation technology, greatly improve the stellite coating performance. With nitrogen ion bombardment sputtering deposition layer and ion beam mixing preparation method of Stellite alloy protection layer properties more excellent.

Using ion implantation technique for surface processing, the modification effect, high efficiency, strong controllability and maneuverability, the advantages of no pollution gradually show strong vitality and superiority. Ion injection and after injection of temperature control can be arbitrary, and in a vacuum, no oxidation, no deformation, no annealing softening occurs, the surface roughness generally no change, can be used as the final process. But look from current levels, ion implantation also has some disadvantages, such as only can injection thin layers, ion can only be straight and no bypass, for complex and has inner hole of the parts can't ion implantation, the cost of the equipment are high, so it is not widely used.

Electrospark Deposition. Electro spark deposition can be used as a means to strengthen the surface. It can be used to strength the surface which has the requirements of wear-resistant, corrosion resistance, the surface oxidation .It can also be used to repair the surface by welding and prepare a variety of special functional coatings. By using the electric spark deposition technique on surface strengthening treatment can prolong the service life of equipment, reduce consumption of resources, which has a high significance on energy conservation and environmental protection [22-24]. Liuwei Zhang comes from Hubei University of Technology, have done an experiment by using the new electric spark deposition equipment, with stellite alloy 6\# as electrode material, H13 steel as base material [25]. The experiment by changing some process parameters during the electro spark deposition process, detecting a series of changes occurred in the sedimentary layer; find out the relationship between the obtained Stellite depositing layer properties and the deposition parameters. According to the experiment, they find electro spark deposition layer and the matrix material between alloy matrix and deposition layer, belonging to the metallurgy combination, very strong, not easy to fall off. The experiments also found that the capacitance parameter have a significant effect on a series performance of Stellite alloy coating. The number and size of defects in the coating is proportional to the size of the capacitance. Wear resistance and thermal fatigue resistance of Stellite coatings deposited small capacitance is obtained with the large capacitance are greater than the effect of deposition. Compared with previous laser processing and electron beam treatment technology, using the electric spark to deposits the Stellite Alloy, we can get higher hardness and wear resistance, and better combination between matrixes. But the disadvantage of electro spark deposition is the thickness of sediment layer is thin, the deposition rate is low and the deposition layer is not uniform. Although the deposition layer will increase with deposition time increased, not unlimited increase, when the time exceeds a certain limit the layer will become thinner. Therefore, the electric spark deposition of Stellite Alloy is not applicable on the surface of large area and complicated shape.

\section{Problems and Prospects}

At present, the domestic study of Stellite alloy treatment process focus on changing the process parameters. Then qualitative research on microstructure, microhardness, corrosion resistance, wear resistance and other properties of multi-layer after treatment, finally points out that these properties are improved. But because each process has its own characteristics, even found out the optimal technique process in one of the treatment process, the preparation of the product in the condition of extremely complex situations still may not be able to meet all requirements. Like the above laser cladding and electron beam cladding, although the Stellite coatings formed is uniform, thickness is big, make up the shortcomings of the electro spark deposition process of thin, but the hardness relative to electro spark deposition is lower. But if some parts need high hardness and thickness of the 
coating, full use of laser and plasma of cladding Stellite alloy powder the required cost is too high [26].

Technology for the processing of single surface has certain limitation. Now, the integrated use of two or more surface technology for composite processing method has been developed rapidly [27-28]. The surface treatment of two or more than two kinds of surface technology are applied to the same work piece, not only can play a variety of surface technology features, and the combination of advantages can better highlight the process use. As of now there have combination surface treated electro spark deposition and laser cladding to research, to explore can produce better superior alloy clad technology. The future will be combined with various processing technology to study the Stellite alloy is a trend.

Another problem is that the domestic research on treatment process of Stellite Alloy, get the conclusion although that could improve the performance of Stellite alloys, and pointed out the limitations of the use process, but they are not to explore the treatment process may cause some side effects. Stellite alloy is a kind of high quality alloy is mainly used in precision parts, once the failure, may cause serious consequences. The domestic should also gradually begin to explore the processing of negative effect in the process.

\section{Conclusion}

As mentioned above, the Stellite Alloy as high temperature carbide has excellent performance, and broad prospects for development. With the continuous development of science and technology, all kinds of intelligent processing technology will be constant innovation. However, for a variety of various processing Stellite alloy process, overlapping seepage analysis is seldom considered. Need the focus after the study was to investigate the possibility of combining different preparation good Stellite stratified, and parameter optimization, in order to find out the process and lower cost of vulnerable to promote manufacturing methods.

\section{Acknowledgments}

This paper was supported by the science and technology research project of Jiangxi Education Department in 2016 with the project name Research on Spreading Prediction of Forest Fire Based on GM $(1,1)$ Model.

\section{References}

[1] Liu Yuzhen, GUI yewei. The properties and application of Stellite alloys (I) [J]. Materials for mechanical engineering, 1992.

[2] Liu Yuzhen, GUI yewei. The properties and application of Stellite alloys (II) [J]. Materials for mechanical engineering, 1992.

[3] Wen Ni. The research about last stage blades of steam turbine mosaic stellite alloy slices to improve corrosion resistance [J]. Tianjin electric power technology, 2006.

[4] Chen Xiao, Zhao Longfei, Wang Bitong, Li Xingdong. 2Cr13 last stage blade brazing braze welding stellite alloy slices of rational [J]. Heilongjiang metallurgy, 2014.

[5] Shen Yong, Huang Ningkang, Wang Minghua. Using DC magnetron sputtering deposited stellite alloy thin films [J]. Journal of Sichuan University (NATURAL SCIENCE EDITION), 1988.

[6] Huang Ningkang, Wang Minghua, Guo Huacong, Peng Xiufeng. The main elements of RF magnetron sputtering thin Stellite film deposited measurement [J]. Journal of Sichuan University (NATURAL SCIENCE EDITION). 
[7] Huang Ningkang, Wang Minghua, Shen Yong, Guo Huacong, Wang Xianen. Thin film by magnetron sputtering of Stellite Alloy [J]. Electric power, 1987.

[8] Zhu Kai, que mefo, Zhu Zhiyuan, Dai Anlun, Chen Hui, Niu wenming. Effects of stellite alloy plasma arc surfacing welding layer parameters of dilution rate of [J]. Welding technology, 2014.

[9] Huang Lan, Pu Yong, Qiu Shulin. The plasma spray welding valve core components in turbine stellite alloy welding application [J]. Dongfang Electric review, 2007.

[10] Qian miaogen, Guo Xingwu. Modern surface engineering [M]. Shanghai Jiao Tong University press, 2012.

[11] Huang Ningkang. The surface of the blade stellite alloy complex of laser treated layer [J] Turbine Technology, 1986.

[12] Qi Keqiang. Study on the process of laser surface cladding mode stellite alloy powder hot [J]. Hot working technology, 2013.

[13] Zhao Wenyu, Lu Feng gui, Li Zhuguo, Wang Dong, Wang Xiaojuan, Liu Xia, Yang Renjie. Study on high cycle fatigue behavior of $17-4 \mathrm{PH}$ stainless steel by laser surface cladding Stellite6 alloy coating [J]. Chinese laser, 2014, 10:131-136.

[14] Zhong Minlin, Liu Wenjin, Chen Yanxia, Ning Guoqin. Stellite Alloy and WC laser cladding [J]. Transactions of metal heat treatment, 2001.

[15]Bear cloud, Wang Yong, Zhang Kaifeng, Zhang Genqing. Study on Microstructure and properties of laser cladding Stellite6/WC [J]. China surface engineering, 2008.

[16] Wang Dong, Li Zhuguo, Wang Xiaojuan, Yang Renjie, Qiao Shangfei, Liu Xia. Tail wind protection of Stellite 6 alloy laser cladding coating and the influence of mechanical properties [J]. China surface engineering, 2014.

[17] Feng Zhirong, Huang Ningkang. Study on the corrosion resistance layer by electron beam melting stellite alloy composite $[\mathrm{J}]$. Journal of Sichuan University (NATURAL SCIENCE EDITION), 1989.

[18] Huang Ningkang, Wang Xi, Zhang Xiaozhong, Wang Xiaopu. The electron beam melting pressure stellite alloy coatings [J]. Heat treatment of metal spraying, 1989.

[19] Xu Qiao Fu. Application and development of ion bombardment of the surface strengthening technology [J]. Aeronautical Manufacturing Technology, 2001.

[20] Wang Ming, Shi Qingnan, Chen Yongjun. Research progress in modification of material surface ion implantation [J] .Yunnan metallurgy, 2003.

[21] Zhang Tonghe. Surface optimization technology of ion implantation [M]. Beijing:Metallurgical Industry Press, 1993.

[22] Huang Ningkang, Feng Zhirong. Study on corrosion resistance of stellite alloy deposition layer by ion bombardment [J]. Nuclear Techniques, 1992.

[23] Wang Jiansheng , Electric spark deposition and alloying [D]. Kunming University of Science and Technology, 2004

[24] Chen Weiwei, Zhu Ying, Kang Hui, Qu Ping. Electro spark deposition technology research status at home and abroad [J]. Welding \& Joining, 2006.

[25] Wang Jiansheng .Study of spark deposition process and performance of electro deposition layer [J]. Surface technology, 2005. 
[26] Zhang Liuwei, Wang Chunjie, Zhou Xiaoping. Microstructure of H13 steel surface electric spark deposition of Stellite Alloy [J]. Hot Working Technology, 2013.

[27] Wang Jiansheng, Li Gang, Liu Youying, Cheng Rui. Electric spark deposition and laser cladding microstructure and properties of composite coating [J]. Transactions of Materials and Heat Treatment ,2012.

[28] Zhao Bin, Li Zhichao, Li Hewan. Research progress of composite coating technology [J]. Hot working technology, 2010. 